54, 4, pp. 1169-1181, Warsaw 2016

DOI: $10.15632 /$ jtam-pl.54.4.1169

\title{
LOAD DISTRIBUTION IN THE WORM MESHING
}

\author{
HENRYK G. SABINIAK \\ Technical University of Lodz, Lódź, Poland \\ e-mail: henryk.sabiniak@p.lodz.pl
}

\begin{abstract}
The paper presents a theoretical analysis of deflections of circular and rectangular cantilever plates of an elastically built-in edge. The problem has been solved using the finite difference method. A method of determination of load distribution between two plates being in contact along an arbitrary line has been presented. Different shapes of these plates constitute models of mating of cylindrical and globoidal worm meshings. To verify the presented theory of searching for load distributions along the contact lines in these meshings, experimental investigations with the freezing of stresses in the cylindrical worm meshing have been carried out. Directions of investigations that would specify further theoretical considerations over load distribution in globoidal gear meshings have been indicated.
\end{abstract}

Keywords: finite differences, circular plate, cantilever plates, worm teeth, load distribution

\section{Notations}

$A$ - distance of axes, auxiliary constant

$D$ - binding rigidity of plate

$E, \nu$ - Young's modulus and Poisson's ratio

$F, F_{i}$ - force and force acting at point $i$ of contact line, respectively

$F_{i}^{1}\left(r_{i}, \varphi_{i}\right), F_{i}^{2}\left(r_{i}, \varphi_{i}\right)$ - force acting at point $i$ of contact line of plate 1 and 2 , respectively $F_{w}$ - inter tooth force

$K_{r, \varphi_{i}}^{r_{i}, \varphi_{i}}$ - dimensionless parameter of deflection of plate

$R, R_{n}$ - minimum and maximum radius of the plate, respectively

$R_{z}$ - radius of tooth tips of worm wheel

$h$ - plate thickness

$m$ - module

$p$ - screw parameter

$q$ - linear load

$r$ - average radius of circular cantilever plates

$r_{g}$ - radius of globoid of worm wheel

$r_{n}$ - radius of cylinder pitch of worm

$z_{1}$ - number of teeth of worm

$(r, \varphi),\left(r_{i}, \theta, z\right),(x, y, z)$ - polar, cylindrical and rectangular coordinates, respectively

$\alpha_{n}$ - pressure angle

$\beta$ - angle between contact line and rubbing speed vector

$\varphi_{1}$ - angle of rotation of worm

$\omega$ - deflection 
$\omega_{0}(r, \varphi), \omega_{0}^{1}(r, \varphi), \omega_{0}^{2}(r, \varphi)$ - deflection at point of coordinates $(r, \varphi)$ corresponding to total load acting on plate, plate 1 and plate 2 , respectively

$\omega_{i}(r, \varphi)$ - deflection at point of coordinates $(r, \varphi)$ caused by concentrated force $F_{i}\left(r_{i}, \varphi_{i}\right)$ acting on plate

Indices: I,II,III - numbers of the contact lines.

\section{Introduction}

The compactness of construction, silent running and the possibility of obtaining a large transmission ratio on one stage, cause the worm gears to be one of the most popular types of toothed gears.

At the stage of designing cylindrical and globoidal worm gears, there are actually two problems to be dealt with: rubbing speed and load distribution in the meshing.

Great rubbing speeds in the meshing are characteristic for worm gears. This is their negative feature, since they are the source of power losses which, in turn, change into heat during the operation of the gear. This problem is well-known to the constructors of worm gears who can lessen this negative effect while designing by a proper selection of tooth profile geometry, a shift in the profile and the selection of material (Buckingham, 1960; Niemann and Winter, 1983; Crosher, 2002; Dudás, 2005).

On the other hand, load distribution in the worm gear meshing can be considered unknown or hardly known. The knowledge of distribution of load along the contact lines of the mating teeth and the related distribution of stresses in the tooth foot, as well as of the laws governing the above will enable one - through constructional procedures at the design stage - to obtain such a distribution of load that will allow increasing the durability of the meshing or its load-carrying capacity.

The widespread use of rectangular and circular cantilever plates in machines and structures as their real elements, or as physical-mathematical models of real systems, makes it necessary for the designer to become better-acquainted with the deformability of cantilever plates replacing real fragments of machine parts. The present work aims at solving a number of problems concerning the cantilever plate theory, with the special question being their adaptation to globoidal and cylindrical worm meshings.

\section{The basics of the worm meshing theory}

The external surface (tip diameter) of the worm and the surface of the globoid and the external (maximum) diameter of the worm wheel interpenetrate and define the area in which the mating of the worm teeth and the worm wheel takes place. The determination of the area of mating and the contact line will be presented basing on the simplest example from the point of view of meshing geometry, namely for the gear with a worm of the profile of Archimedes teeth. Archimedes worms are characterized by a rectilinear profile in the axial section, while the front section of these worms is in the shape of the Archimedes spiral.

The geometry of such a worm meshing can be described by the formulae (Litwin, 1959)

$$
\begin{aligned}
& {\left[p\left(\Theta-\varphi_{1}\right)-r_{i} \tan \alpha_{n}\right]\left(\cos \Theta \tan \alpha_{n}+\frac{p \sin \Theta}{r_{i}}\right)-r_{i} \cos \Theta=r_{n}} \\
& x=r_{i} \sin \Theta \quad y=r_{i} \cos \Theta \quad z=p\left(\Theta-\varphi_{1}\right)-r_{i} \tan \alpha_{n} \quad p=\frac{m z_{1}}{2}
\end{aligned}
$$

If $\varphi_{1}=$ const, then equations (2.1) define instantaneous contact lines for a given angle of rotation of the worm. The contact between the worm teeth and the worm wheel for a given gear 
takes place only in the area common for both these elements. This area is referred to as a tooth contact area (mashing area). In other words, the tooth contact area (Fig. 1) is a working part of the meshing area limited by the above-mentioned areas of the worm pair (Fig. 1) (Sabiniak, 1996); namely:

- the apex diameter of the worm $d_{z}($ curve $a)$,

- the globoidal area of the worm wheel with the radius $r_{g}$ corresponding to it (curve $b$ ),

- the maximum diameter of the worm wheel $R_{z}$ (curve $c$ ).

(a)

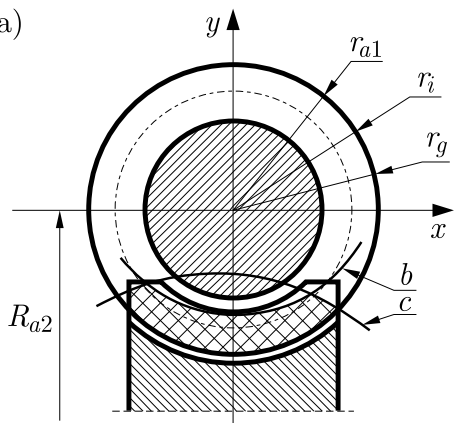

(b)

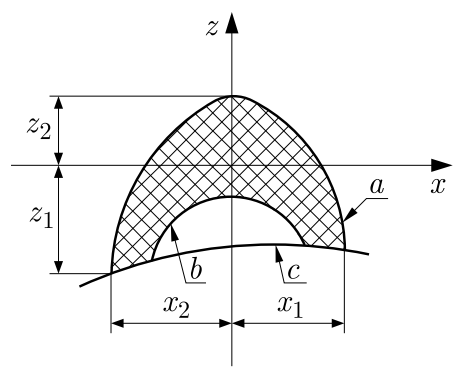

Fig. 1. The area of meshing of the worm pair: (a) in the front section, (b) in the horizontal projection

To define the tooth contact area, one must determine:

- curve a (Fig. 1), assuming $r_{i}=r_{g}$ in equations (2.1), i.e. replace an arbitrary radius of the cylinder with the external radius of the worm,

- curve $b$; then in equations (2.1) one must substitute $r_{i}=r_{g}$, and add the globoid equation

$$
(x+A)^{2}-\left(A-\sqrt{r_{g}^{2}-y^{2}}\right)^{2}+z^{2}=0
$$

- curve $c$, by considering simultaneously equations (2.1) and the equation of the external cylinder of the worm wheel

$$
z^{2}+\left(A+r_{i} \cos \Theta\right)^{2}=R_{z}^{2}
$$

The tooth contact area and the course of the contact lines for the established value of the angle $\varphi_{1}$ is shown, by way of example, in Fig. 1 (Sabiniak, 1996).

\section{The characteristics of the contact lines in the worm meshing}

The course of the contact lines in the tooth contact area has a significant effect on the operating parameters of the gear.

Modern tendencies in the construction of worm gears aim at finding such a position for the contact lines as to let angle $\beta$, which they form with the vector of the rubbing speed (Fig. 2), approach $90^{\circ}$. Favorable conditions are then created for the formation of an oil film, and the amount of heat in the mashing is simultaneously reduced. In addition, the shape of the contact lines has a decisive effect on the load distribution (which will be presented further). In turn, the course of the contact line largely depends on the profile of the mating surfaces of the teeth and the worm wheel addendum modification coefficient. In principle, there are two groups of profiles:

- rectilinear profiles,

- curvilinear profiles. 
The first group comprises all worms made using a rotational tool of a rectilinear profile or those that have a straight line in any section. The following profiles can be distinguished here:

- Archimedes profiles,

- rectilinear in the section perpendicular to the helix on the tooth reference cylinder,

- evolvent,

- conoidal.

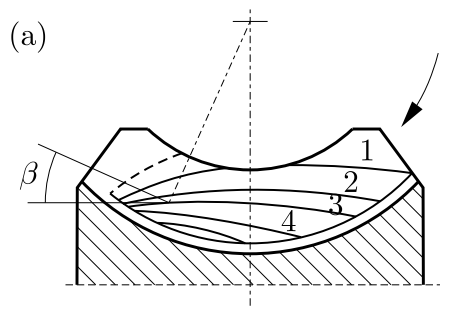

(b)

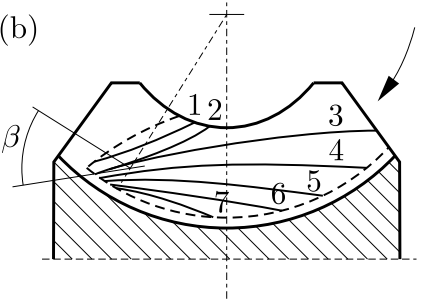

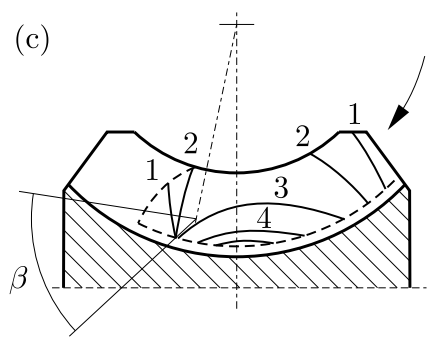

(d)

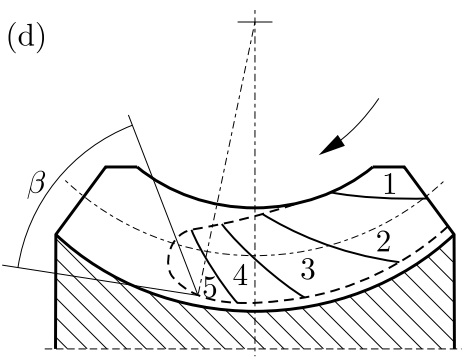

(e)

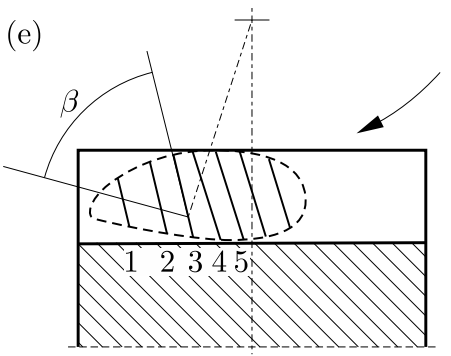

Fig. 2. The course of the contact lines and values of the angle in gears with the following worms:

(a) cylindrical of the profile of Archimedes teeth, (b) cylindrical of the evolvent teeth profile, (c) cylindrical of the circular-concave teeth profile, (d) cylindrical of the circular-convex teeth profile, (e) globoidal

The contact of the teeth of the worm and the worm wheel in the gears with a rectilinear worm is a contact of two convex surfaces, which results in the formation of great inter tooth pressures. Then, small equivalent radii of the curvatures of the profiles along the contact line occur.

In the group of curvilinear profiles, the following worms can be distinguished:

- of a circular-concave profile in the axial section, Cavex,

- of a circular-convex profile in the axial section.

Of the profiles discussed so far, worm gears with a globoidal worm (Fig. 2e) have the best conditions for the formation of an oil film. Moreover, at least one more pair of teeth mates in the case of cylindrical worm gears. The technology of making globoidal worms requires making use of special machines. They are also troublesome to check. However, their operating properties are superior to all the ones discussed hitherto.

\section{Load distribution along the contact line in the worm meshing}

While solving the problem of load distribution along the contact lines in the worm meshing, it has been assumed that the tooth of the worm or the worm wheel is a cantilever plate having one edge built-in elastically (Sabiniak, 1992). The bar between the plates illustrates the contact line and ensures the transfer of load from one plate - a tooth - to another along the contact line required (Figs. 3a and 3b). 
(a)

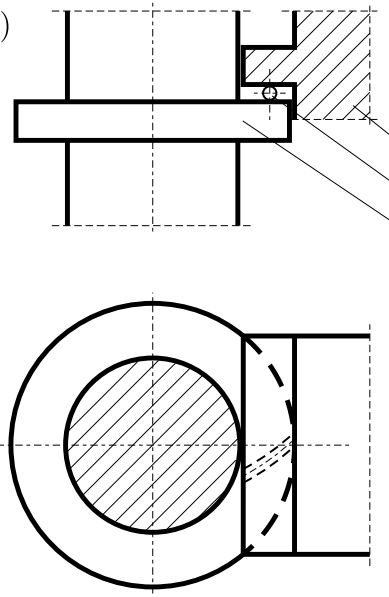

(b)
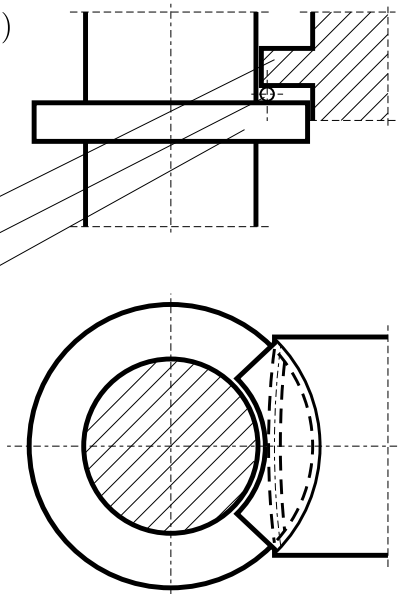

Fig. 3. (a) The model of meshing of the globoidal worm gear: 1 - tooth of the cylindrical wheel, 2 - bar illustrating the contact line, 3 - worm tooth; (b) the model of meshing of the cylindrical worm gear:

1 - worm wheel tooth, 2 - bar illustrating the contact line, 3 - worm tooth

Prior to the determination of load distribution, the question of deformability of the plate, depending on its geometric parameters and material constants, has been solved by forming matrices of rigidity of the plates (Sabiniak, 1982, 1992). Calculations of the strains (or more precisely, of the dimensionless parameters of deflection of the plate $\left.K_{r, \varphi}^{r_{i}, \varphi_{i}}\right)$ have been made for each type of the plate (circular cantilever plate and a circular plate in the shape of a circular sector) by applying the concentrated force to appropriately selected places. These appropriately selected places are nodes of the net superimposed on the plates, through which contact lines pass.

Basing on these results, one could start searching for load distribution along the contact line (Fig 3 and 4), assuming at the same time that:

- the continuous load will be replaced with a number of concentrated forces (Sabiniak, 1992; Umezawa et al., 1969; Jaramillo, 1950)

$$
F_{i}=\int_{l_{i-1}}^{l_{i}} q d l
$$

- the total strain of the plate is a superposition of the previously obtained deflections from particular, independently acting concentrated forces (Sabiniak, 2012; Sabiniak and Woźniak, 1988)

$$
\omega_{0}(r, \varphi)=\sum_{i=1}^{i=n} \omega\left(r_{i}, \varphi_{i}\right)
$$

- the algebraic sum of deflections of both plates along the contact line is a constant value

$$
\omega_{0}^{1}(r, \varphi)+\omega_{0}^{2}(r, \varphi)=\omega=\text { const }
$$

- the forces of reciprocal interaction in the corresponding points along the contact line of both plates are equal to each other

$$
F_{i}^{1}\left(r_{i}, \varphi_{i}\right)=F_{i}^{2}\left(r_{i}, \varphi_{i}\right)
$$


- the sum of forces acting in particular nodes of the contact line must be equal to the inter tooth force

$$
F_{w}=\sum_{i=1}^{i=n} F_{i}\left(r_{i}, \varphi_{i}\right)
$$

\section{The mating of circular cantilever plates and those in the shape of the circular sector}

The deflection of the plate at the point of coordinates $(r, \varphi)$, caused by the action of the concentrated force applied at the point of coordinates $(r, \varphi)$, has the form

$$
\omega_{i}(r, \varphi)=\frac{h^{2}}{\pi D} K_{r, \varphi}^{r_{i}, \varphi_{i}} F_{i}\left(r_{i}, \varphi_{i}\right)
$$

where $K_{r, \varphi}^{r_{i}, \varphi_{i}}=f\left(R, R_{1}, R_{n}, E, F, F_{i}, F_{w}, h, r_{i}, v, \varphi, \varphi_{i}\right)$ have been taken from work (Sabiniak, 1992; Umezawa et al., 1969; Jaramillo, 1950).

The total deflection of the plate at the point of coordinates $(r, \varphi)$ will be the sum of deflections from particular forces acting on the plate

$$
\omega_{0}(r, \varphi)=\frac{h^{2}}{\pi D} \sum_{i=1}^{i=n} K_{r, \varphi}^{r_{i}, \varphi_{i}} F_{i}\left(r_{i}, \varphi_{i}\right)
$$

When the contact lines do not pass exactly through the nodes of the net, interpolation is performed. If the point of the contact line, e.g. $K$ (Fig. 4) is on the line of the net between two nodes, them the dimensionless parameter of deflection of the plate $K_{K}$ for such a point is determined from the dependence $K_{K}=a_{2} K_{A}+a_{1} K_{B}$, where $a_{1}+a_{2}=1$. If the point of the contact line $L$ is not on any line of the net (Fig. 4), then the dimensionless parameter of deflection of the plate of such a point is determined from the dependence $K_{L}=c_{2}\left(b_{2} K_{E}+b_{1} K_{D}\right)+c_{1}\left(b_{2} K_{B}+b_{1} K_{C}\right)$, assuming that $b_{1}+b_{2}=c_{1}+c_{2}=1$.

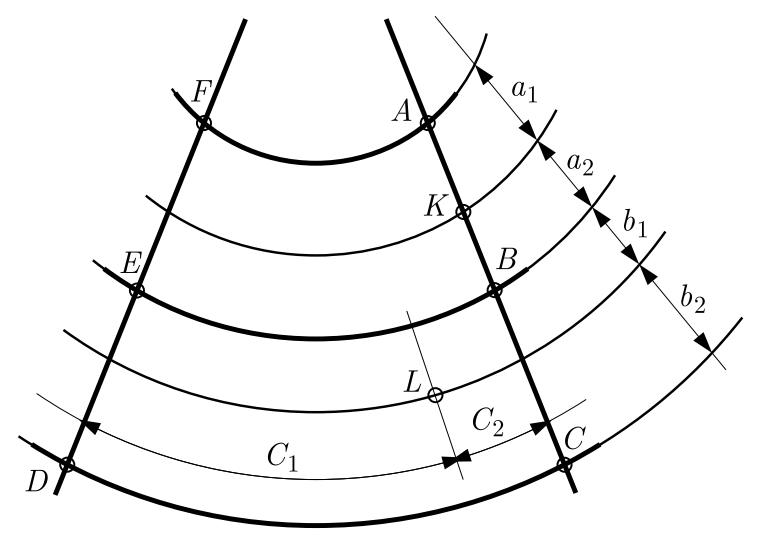

Fig. 4. The interpolation of the deflections of the plate points that do not coincide with the nodes

For the dimensionless parameters of deflections of the plate in particular points of the line of contact (5.2) thus calculated, condition (4.3) can be employed. If the number of the concentrated forces replacing the constant load, equal to the nodes lying on the contact line is $i$, then following the application of condition $(4,3)$ - a system of $i$ linear equations containing $i+1$ unknowns (particular forces in the nodes and the total deflection) will be obtained in each node. The missing equation is obtained by equating the sum of force acting in particular nodes of the contact line to the resultant force acting on the plate (4.5). 
In the toothed gears it results from the action of the inter tooth force (Sabiniak, 1982, 1992; Umezawa, 1974). The course of calculation procedure will be shown using an example.

\section{A numerical example}

Find the distribution of the inter tooth force between two cantilever plates:

- the circular (of steel) with material constants $E_{1}=2.06 \cdot 10^{11} \mathrm{~N} / \mathrm{m}^{2}$ and $\nu_{1}=0.3$ and dimensions $R_{n 1}=60 \mathrm{~mm}$ - the external radius of the circular plate - of the worm convolution apexes, $R_{1}=38 \mathrm{~mm}$ - the internal radius of the circular plate - of the bases of the worm convolution and $h_{1}=7.5 \mathrm{~mm}$ - the thickness of the circular plate equal to the average thickness of the worm teeth;

- in the shape of the circular sector (of bronze) with material constants $E_{2}=10^{11} \mathrm{~N} / \mathrm{m}^{2}$ and $\nu_{2}=0.35$ and the dimensions $R_{2}=62 \mathrm{~mm}$ - the external radius of the plate in the shape of the circular sector - the radius of the bases of the worm wheel teeth, $R_{2}=40 \mathrm{~mm}$ - the internal radius of the plate in the shape of the circular sector - the radius of apexes of the teeth of the worm wheel and $h_{2}=12 \mathrm{~mm}$ - the thickness of the plate in the shape of the circular sector equal to the average thickness of the worm wheel tooth contacting each other along the line shown in Fig. 5 and designated as number I. The deflections of the circular cantilever plate made of steel (the worm) in particular points of the contact line amount to

$$
\begin{aligned}
& \omega_{50,-45}^{1}=\frac{h_{1}^{2}}{\pi D_{1}}\left(K_{50,-45}^{50,-45} F_{50,-45}+K_{50,-45}^{50,-40} F_{50,-40}+\ldots+K_{50,-45}^{50,40} F_{50,40}+K_{50,-45}^{50,45} F_{50,45}\right) \\
& \omega_{50,-40}^{1}=\frac{h_{1}^{2}}{\pi D_{1}}\left(K_{50,-40}^{50,-45} F_{50,-45}+K_{50,-40}^{50,-40} F_{50,-40}+\ldots+K_{50,-40}^{50,40} F_{50,40}+K_{50,-40}^{50,45} F_{50,45}\right) \\
& \vdots \\
& \omega_{50,40}^{1}=\frac{h_{1}^{2}}{\pi D_{1}}\left(K_{50,40}^{50,-45} F_{50,-45}+K_{50,40}^{50,-40} F_{50,-40}+\ldots+K_{50,40}^{50,40} F_{50,40}+K_{50,40}^{50,45} F_{50,45}\right) \\
& \omega_{50,45}^{1}=\frac{h_{1}^{2}}{\pi D_{1}}\left(K_{50,45}^{50,-45} F_{50,-45}+K_{50,45}^{50,-40} F_{50,-40}+\ldots+K_{50,45}^{50,40} F_{50,40}+K_{50,45}^{50,45} F_{50,45}\right)
\end{aligned}
$$

Identical dependences must, of course, be written for the cantilever plate in the shape of the circular sector made of bronze (the worm wheel). From the symmetry of the system occurring for this case, it can be clearly seen that

$$
\begin{aligned}
& F_{50,-45}=F_{50,45} \\
& F_{50,-40}=F_{50,40} \\
& \vdots \\
& F_{50,-5}=F_{50,5} \\
& F_{50,0}=F_{50,0}
\end{aligned}
$$

Expression (6.1) and the one obtained in the similar manner for the plate made of bronze, must be substituted into condition (4.3), taking at the same time dependences (4.4) and (6.2) into account. Then, while replacing the continuous load along the contact line with the concentrated forces, 19 in number, and making transformations, a system of 10 linear homogeneous equations will be obtained 


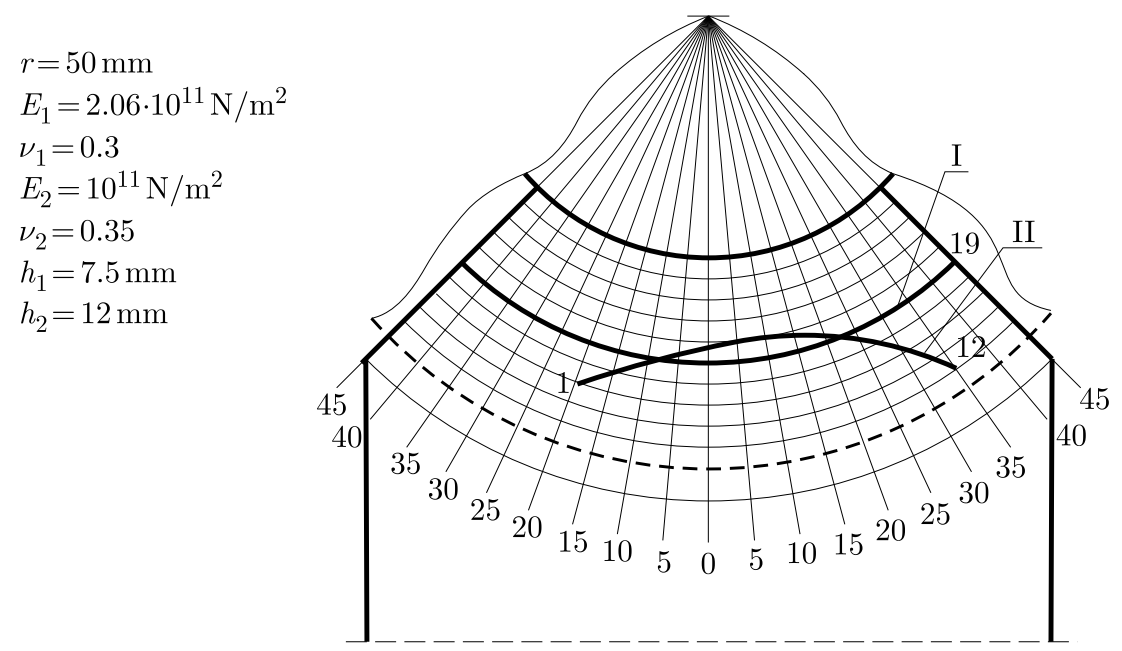

Fig. 5. The net of division and the contact lines in the cylindrical worm meshing

$$
\begin{aligned}
& \omega_{50,-45}^{1}+\omega_{50,-45}^{2}-\omega=0 \\
& \omega_{50,-40}^{1}+\omega_{50,-40}^{2}-\omega=0 \\
& \vdots \\
& \omega_{50,-5}^{1}+\omega_{50,-5}^{2}-\omega=0 \\
& \omega_{50,0}^{1}+\omega_{50,0}^{2}-\omega=0
\end{aligned}
$$

Substituting the values of $K_{r, \varphi}^{r_{i}, \varphi_{i}}$ calculated in works (Sabiniak, 1982, 1986, 1992) and adding condition (4.5), the following system of equations is obtained, allowing one to determine forces in particular nodes of the net.

The calculation results are graphically presented in Fig. 6.

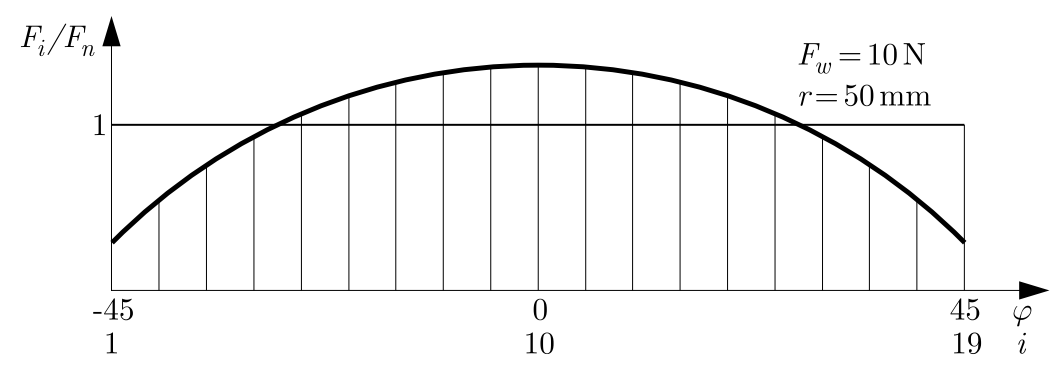

Fig. 6. The load distribution for contact line I according to Fig. 5

\section{The mating of circular and rectangular cantilever plates}

While searching for load distribution along the contact line in the globoidal meshing, the same formulae (4.1)-(5.2) are applied.

Additional calculations are required only in the case of making linear equations for contact line I (Fig. 7). Contact line I does not pass precisely through the net nodes. In this case, interpolation is also used for finding the coefficients $K_{r, \varphi}^{r_{i}, \varphi_{i}}$, (Fig. 4). The procedure described refers to the calculations carried out for both the polar and rectangular coordinates.

It has been decided that - for the rectangular plate - the nodes would occur where contact line I would cross horizontal lines of the net. Following such an assumption, with the interpolation 


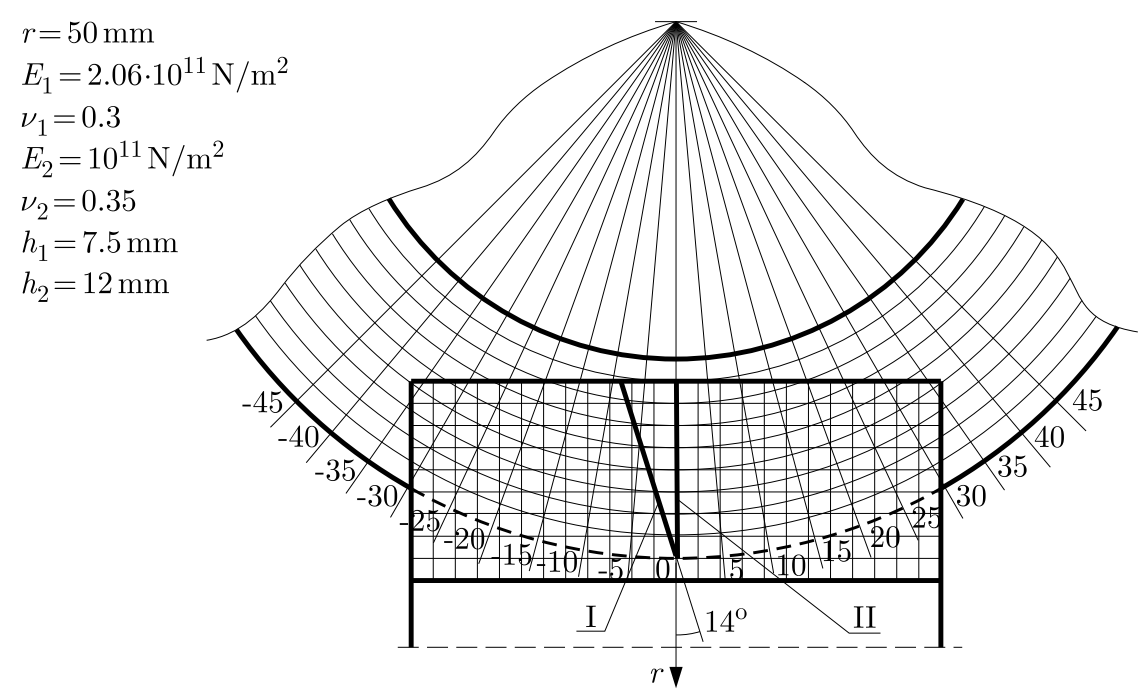

Fig. 7. The net of division and the contact lines in the globoidal worm meshing

of the coefficient $K_{r, \varphi_{i}}^{r_{i}, \varphi_{i}}$ for a plate in the shape of the circular sector, four adjacent points for the net of polar coordinates are taken into consideration, as described in Section 4, and only two adjacent points for the net of rectangular coordinates.

The shape and designations of the contact lines are shown in Fig. 7, while the load distributions corresponding to particular contact lines in Figs. 8. The distributions of load obtained for these lines do not differ radically, which results from a slight difference in the course of these lines.

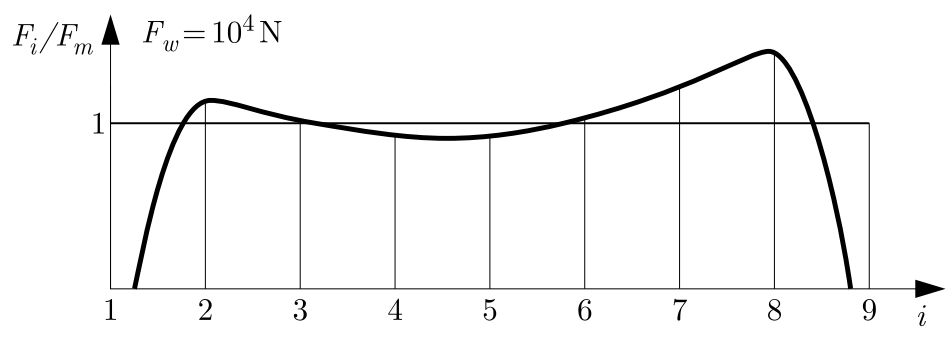

Fig. 8. The load distribution for contact line I according to Fig. 9

\section{The results of theoretical and experimental investigations}

Figure 9 presents the course of the contact lines on particular teeth of the worm wheel in the worm gear studied, obtained during experimental investigations.

Figure 10 presents the stresses that occurred in the cylindrical worm meshing. The concentration of stresses in particular points of contact for particular teeth is characteristic here.

Figure 11 is a diagram of changes in contact stresses along the contact line.

The contact line for tooth 6 (Fig. 9) can be equated to contact line I in Fig. 5. Here is a slight divergence between the course of the contact line in the tooth contact area. Thus, the stresses of Fig. 11 can be compared with the load distribution in Fig. 6. As there are principally different quantities "stress" and "force" in these figures, there is no justification for comparing them with respect to absolute values. However, as has been mentioned at the beginning of the paper, experimental investigations aimed at the qualitative verification of the theory of load distribution along the contact line (are not?) discussed in the present work. 
(a)

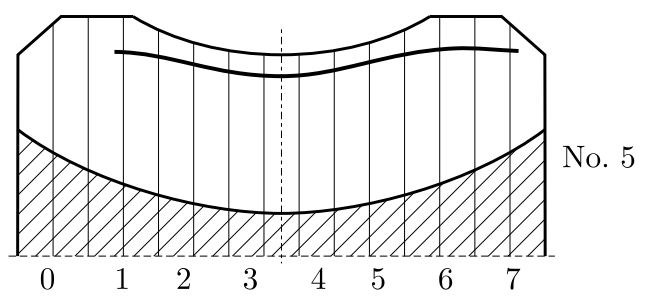

(b)

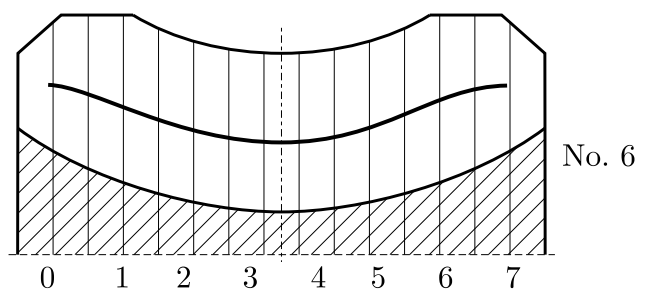

(c)

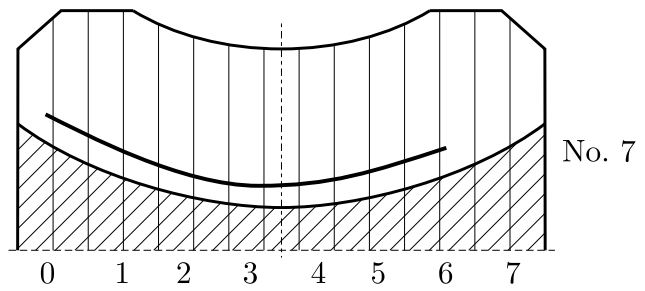

Fig. 9. The real contact lines in the worm gear under investigation: (a) for the worm wheel tooth entering the meshing, designated by number 5, (b) for the central worm wheel tooth being in the meshing, designated by number 6 , (c) for the worm wheel tooth leaving the meshing, designated by number 7

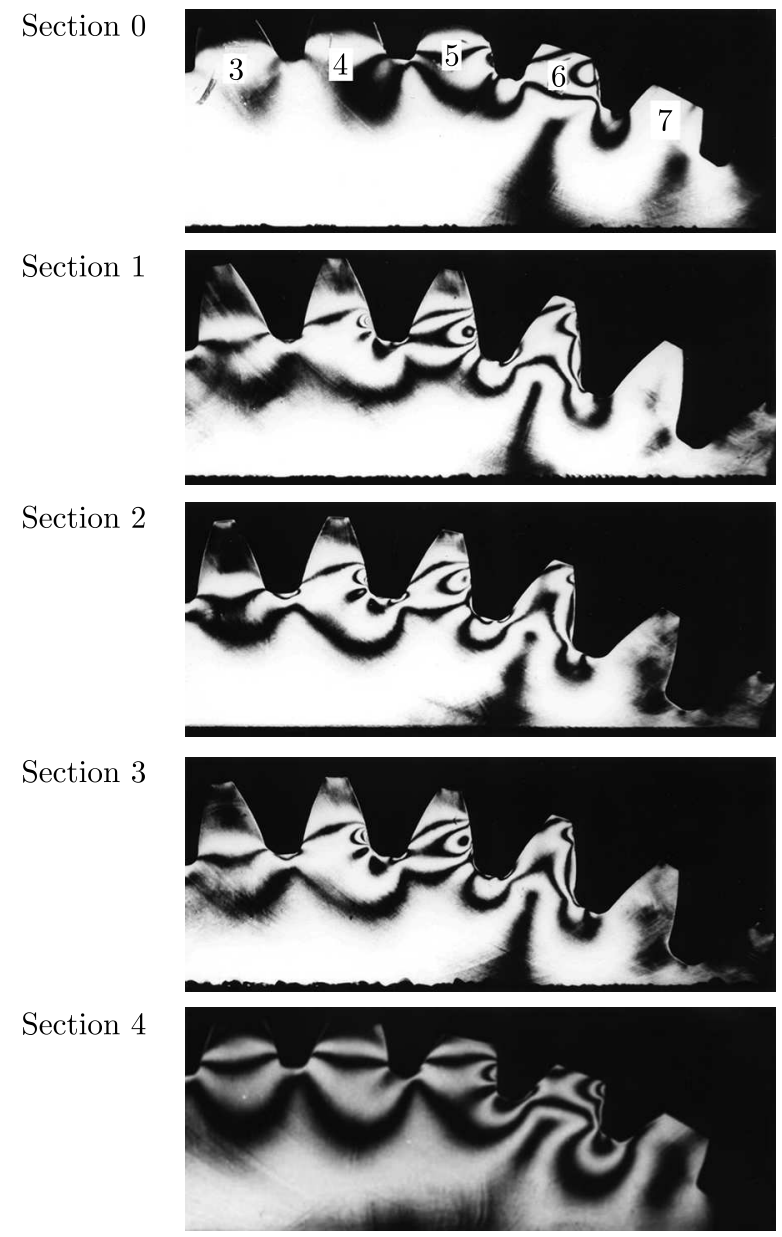

Fig. 10. The state of stresses in the cylindrical worm meshing in particular sections of the worm wheel according to Fig. 9 


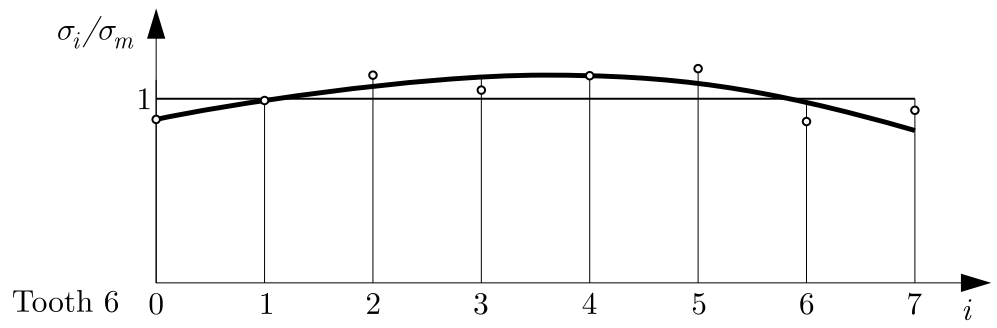

Fig. 11. The changes in the contact stresses along the contact line in the cylindrical worm meshing

In other words: if the load changes, its character along the contact line decreases, increases, rapidly concentrates, fades, etc. The change in contact stresses should be of the same character in the same places. The stresses in the tooth foot should assume a similar, although slightly milder, character. When comparing Figs. 11 and 6 with this respect, a similarity of the course of load distribution and contact stresses along the contact lines discussed can be easily observed.

\section{Directions of further investigations}

The present work constitutes solely one of the problems which, as will be presented below, has not been fully dealt with. It is one in the whole range of problems related to globoidal meshings.

As has been mentioned in the works (Sabiniak, 1982, 1986, 1992; Hayman and Sabiniak, 1986; Predki, 1991), owing to its specificity, globoidal meshings possess a serious difficulty in scientific studies, both theoretical and experimental. The skill of finding load distribution along any contact line is a fundamental problem, the knowledge of which allows passing on to further investigations in this field. The use of the knowledge and the load distribution along the contact line is connected to finding an answer to the question: how will the mating surfaces of the teeth be deformed under such a load? The problem of hertzian pressures along with the Bielajew problem (Niemann and Winter, 1983) is currently relatively well-known and there seems to be no difficulty in the adaptation of this knowledge to globoidal meshings.

The next step, which appears the obvious thing to do, is the introduction of an elastothermohydrodynamic oil film between the mating teeth. Such attempts are already in progress.

If the oil film is to be considered, then the next step is the introduction of temperature into the meshing and finding its distributions, both in the oil film and in particular mating teeth. It is clear that a great amount of heat is released during the operation of worm gears. Thus, the question arises what portion of the energy is changed into heat, and how it is dissipated. There are works concerning basic studies on plates where the thermal problem has been solved. However, temperature has been treated as a boundary condition there (Jaramillo, 1950; Timoshenko and Woinovsky-Krieger, 1959; Umezawa et al., 1969; Kacczkowski, 1980). The solutions in thermal investigations of plates thus presented are difficult to interpret physically and cannot be practically applied in the problem discussed. The attempts at tackling the heat problem of worm gears aim rather at operational uses than at elucidating the phenomena occurring in the worm meshing.

The development of numerical methods in mathematics, particularly of the finite element and finite difference methods (Sabiniak, 2012), allows finding load distributions in worm meshings when the thickness of the tooth, as a plate, changes arbitrarily, which will probably be the next stage in further research.

However, the problem of distribution of the load into particular pairs of mating teeth has yet not been solved (Litwin, 1959; Niemann and Winter, 1983; Crosher, 2002; Dudás, 2005).

Either the problem of strain of the worm as a shaft has not been solved. 
The deflections occurring in bearings and strains of the body should not be either forgotten (Sabiniak and Woźniak, 1988).

All the above questions, as well as problems waiting to be solved, are static problems. One can only realize the difficulties of the subject presented if motion is introduced.

\section{Summary}

Basing on the presented theoretical considerations and experimental investigations, as well as on the results obtained, the following conclusions emerge:

1. The finite difference method used with regard to the search for deflections of cantilever plates under arbitrary normal load offers in the solutions of these plates a very good accuracy (Figs. 4-11). In particular, if boundary conditions are carefully selected, then the divergence between the theoretical and experimental results are not greater than $14 \%$.

2. The theoretical and experimental results obtained are in good agreement both for cylindrical and globoidal worm gears, which confirms the rightness of the presented theory of searching for load distributions in the worm meshing (Sabiniak, 2007).

3. The experimental method selected for the verification of the theory presented here should be considered fully justified, although it has already been used for seeking the state of stresses in the meshing. Its application for the presentation of the state of stresses in the worm meshing only confirms the versatility of this method.

4. The results obtained indicate that the existing method of finding the radial course of the contact lines in the tooth contact area (Niemann and Winter, 1983; Litwin, 1959; Crosher, 2002; Dudás, 2005) must be verified. It seems that the method of obtaining the radial course of the contact lines in the tooth contact area by using a great addendum modification in the profile and circular profile of the meshing, at the cost of decreasing the tooth contact area, and first of all, the length of the contact lines occurring simultaneously in the tooth contact area, seems unjustified. A greater length of the contact line significantly reduces the local stress of the materials and generally of the mating teeth thereby improving the load-carrying capacity of the gear and its resistance to wear. Hence, the durability and efficiency of the meshing will increase with length of the contact line in the tooth contact area. It should be remembered, however, that the "straightening" of the contact lines in the tooth contact area impairs, in turn, conditions for the formation of an oil film.

5. The above conclusions show that globoidal worm gears are superior to cylindrical worm gears both with respect to their load-carrying capacity and life, which is nothing new. The above results have again confirmed this truth. The problem that remains is the cost of such a worm in relation to e.g. $1 \mathrm{KW}$ of the power transmitted. This is not so obvious.

6. A change in the character of the course of the contact line in the tooth contact area also changes radically the character of the load distribution.

7. The results obtained are to be used for the modification of the profile of the teeth in order to "equalize" the existing load distribution along the contact line.

\section{References}

1. Buckingham E., 1960, Design and Manufacture of All Resection Worm and Spiral Gear Drives, The Industrial Press, New York

2. Crosher W.P., 2002, Design and Application of the Worm Gear, ASME, USA

3. DudÁs I., 2005, The Theory and Practice of Worm Gear Drives, Elsevier 
4. Haymann J., Sabiniak H.G., 1993, Bestimmung der Spannungen in der Schneckengetriebeverzahnung mit Hilfe des Spannungsoptischen Erstarrungsverfahren, Zeszyty Naukowe Politechniki Eódzkiej, Mechanika, 83, 103-122

5. Jaramillo T.J., 1950, Deflections and moments due to a concentrated load on a cantilever plate of infinite length, Journal of Applied Mechanics, March, 67-72

6. KąCzkowski Z., 1980, Plates - Static Calculations (in Polish), Arkady, Warszawa

7. Litwin B.L., 1959, Teoria zubczatych zaceplenii (in Russian), Izd. Nauka, Moskwa

8. Niemann G., Winter H., 1983, Maschinenelemente, Band III, Springer-Verlag, Berlin, Heidelberg, New York, Tokio

9. PREDKI W., 1991, Stand der Schneckengetriebeentwicklung, Konstruktion, 43, 233-238

10. SABINIAK H.G., 1982, Load distribution along the line of contact of two cantilever plates as models of thhet of a toothead gear (in Polish), Zeszyty Naukowe Politechniki Eódzkiej, Mechanika, 64, 71-82

11. SABiniak H.G., 1986, Load distribution between two cantilever plates contacting each other along a straight line (in Polish), Zeszyty Naukowe Politechniki Eódzkiej, Mechanika, 70, 31-40

12. SABINIAK H.G., 1992, Lastverteilung längesder Berührungslinie zweier Kragplatten, betrachtet als Modell für die Zähne eines Zylinder- und Globoidschneckengetriebes, Technische Universität, Chemnitz, Dissertation B

13. Sabiniak H.G., 1996, Real meshing zone in worm gear (in Polish), Przeglad Mechaniczny, 20, 10-12, 18-20

14. Sabiniak H.G., 2007, Load Capacity and Durability of Worm Gears (in Polish), Monografie Politechniki Łódzkiej

15. Sabiniak H.G., 2012, The method of finite differences in solutions concerning circular plate, Key Engineering Materials., 490, 305-311

16. SABiniak H.G., Woźniak K., 1988, Operating properties of the worm gear with a cavex worm and of circular-convex profil, Conference of Gearing, Zhengzhou, China

17. Timoshenko S., Woinowsky-Krieger S., 1959, Theory of Plates and Shells, Mc Grew Hill Book Company Inc., New York, Toronto, London

18. UmezawA K., 1974, The meshing test on helical gears under load transmission, Bulletin of the Japan Society of Mechanical Engineers, 17, 112

19. Umezawa K., Ishikawa J., Hayashi K., 1969, Deflections due to a concentrated load on a cantilever thick plate of finite length gears, Bulletin of the Japan Society of Mechanical Engineers, $\mathbf{1 2}, 53$ 